Jurnal Teknologi, 38(D) Jun. 2003: 67-78

(C) Universiti Teknologi Malaysia

\title{
COMPUTER-BASED MONITORING SYSTEM FOR ANALYSING SURFACE LEAKAGE CURRENT IN AN IEC 587 TEST SET-UP
}

\author{
MD AFENDI MD PIAH ${ }^{1} \&$ AHMAD DARUS ${ }^{2}$
}

\begin{abstract}
Surface discharge is a common electrical discharge that normally occurs on outdoor insulators under wet and contaminated conditions. The dry-band discharge exists because of the leakage current that flows on the insulator surface. Measuring the surface leakage current can identify the performance of insulating materials as well as the characteristics of discharges. A computer-based on-line monitoring of surface leakage current is found to be more practical due to the long period of time taken in conducting the laboratory test. This paper describes the design and development of a leakage current monitoring system associated with surface tracking and erosion resistance test set-up of IEC 587. The LabVIEW software package is used to develop a measurement program for recording and analysing leakage current signals. Experimental works have shown the capability of the developed system in detecting the performance of insulating materials as well as identifying the characteristics of the surface discharges. Besides the on-line monitoring function, a data storage feature is also added to the system to allow for further analysis.
\end{abstract}

Key words: Surface discharge, leakage current, signal analysis, Virtual instrument program

\subsection{INTRODUCTION}

Insulation plays an important role in order to determine the performance of an electrical power apparatus. A good insulation system will give better design and performance of the apparatus. Today, polymeric insulators (PI) are widely being used in both the distribution and transmission voltage ranges and are steadily capturing a wider share of the market. The tremendous growth in the applications of PI is due to their advantages over the traditional insulators such as porcelain and glass. These advantages are low surface energy, lightweight, higher mechanical strength to weight ratio, resistance to vandalism and better performance in the presence of wet and contamination [1].

The development or use of PI, especially electrical insulation, requires testing of the polymer's physical parameters. The polymer properties take into consideration the whole range of physical parameters, including mechanical, optical, electrical and thermal as well as degradation, non-destructive testing, and tests for processability. The long-term ability of a polymer compound to perform as a high voltage insulator is assessed by means of design test, which involves accelerated test and normal aging test. The analysis of standardized and non-standardized test methods for PI materials applied for high voltage insulation is described in [2].

$\overline{1 \& 2}$ Institute of High Voltage and High Current, Faculty of Electrical Engineering, Universiti Teknologi Malaysia, 81310 Skudai, Johor. 
In order to determine the performance of PI, many measurement techniques are developed for the surveillance and diagnostics of the insulators. The most widely used methods for insulator performance monitoring are the equivalent salt deposit density (ESDD), the surface conductance, the leakage current, air pollution measurements, optical measurements and non-soluble deposit density. The main factors influencing the PI performance are loss of hydrophobicity and material aging under electrical and environmental stresses. The degradation process can develop significantly on the insulator surfaces, because of appearing leakage currents (LC) and discharges. For this reason, measurements of LC are often performed in order to evaluate the performance and degree of aging of polymeric surfaces in laboratory and field studies. Many test methods are presently being used for testing the insulator performance, and information of LC measurement methods on several tests are discussed in [3].

In this paper, a practical on-line surface LC monitoring system associated with IEC 587 test set-up is developed to investigate the characteristics of LC waveforms and their frequency spectrum under surface discharge activity of the polymer insulating materials. The results of $\mathrm{LC}$ analysis from the experimental works are presented in graphical forms using LabVIEW software package.

\subsection{LEAKAGE CURRENT PHENOMENON}

The leakage current (LC), which is driven by the source voltage and collected at the grounded end of the insulator, provides much useful information on describing the state of a contaminated insulator. The analysis of LC is investigated based on LC waveforms and their frequency spectrum characteristics. It is found that the magnitude and harmonic contents of the LC, and discharge duration are significantly different during the portion of the test where there is no visible degradation, as compared to their values at the onset of visible degradation [4]. These findings could be used for early failure detection of high voltage devices for outdoor insulation.

When the insulator is wet, a resistive surface LC flows, which is generally many orders of magnitude higher than the capacitive current in the case of dry insulators. The LC results in non-uniform heating of the contamination layer due to the nonuniform resistivity of the conducting film. This eventually causes dry-bands to be formed at narrow sections of the insulator where the surface LC density is highest. The resistive LC of the insulator then decreases or may even disappear because of the high resistance of the dry-bands. The voltage distribution along the surface of wet contaminated insulator is very non-uniform when a dry-band is formed in series with conductive film. Since almost the whole voltage applied across the insulator appears across the high resistive dry-band, this can result in breakdown of the air above the dry-band. The LC along the insulator can be detected only when the power frequency energizing voltage exceeds a certain positive or negative voltage threshold [5]. The LC becomes non-zero and follows the power frequency waveshape as soon as the energizing voltage is above the threshold value. 
The electrical discharges will cause the high thermal spots to be developed at the arc root and thus leading to gradual erosion of the insulator surface. These repeated discharges burn the insulator surface to create carbonized regions, and so called "surface tracking". Complete breakdown of the insulation surface generally follows when a conducting carbon path propagates to the extent that the remaining insulation is incapable of withstanding the system voltage [6].

Analysis of LC through the observation of different waveshapes and frequency spectrum characteristic could indicate the condition of the insulator surface during the test. It is reported that the transition of LC waveforms, until flashover occurs is classified into different stages and that a threshold exists by which the occurrence of flashover can be predicted [7]. The possibility of flashover occurrence becomes higher when the magnitudes and harmonic contents of the prominent components exceed a particular level.

\subsection{SURFACE TRACKING AND EROSION TEST [IEC 587]}

The IEC 587, popularly known as the Inclined-Plane Tracking (IPT) is one of the major methods for evaluating tracking and erosion resistance of polymeric materials for outdoor insulation. This test attempts to simulate the tracking behavior in a controlled manner in order that comparisons can be made of the tracking resistance of different insulating materials. The tracking test evaluates the relative ability of an insulating material to withstand the action of electrical discharges on the surface, which are similar to those that may occur in service under the influence of environmental stresses. The major electrical equipment, contaminant solution used, equi pment installation as well as the procedures to conduct the test are described in [8]. Figure 1 shows the schematic circuit diagram for surface tracking and erosion test.

Two test methods that are used for evaluating surface tracking resistance of insulating materials are known as 'stepwise tracking voltage' and 'time to track' [8]. When the sample is energized with high voltage stress under wet contaminated condition, the

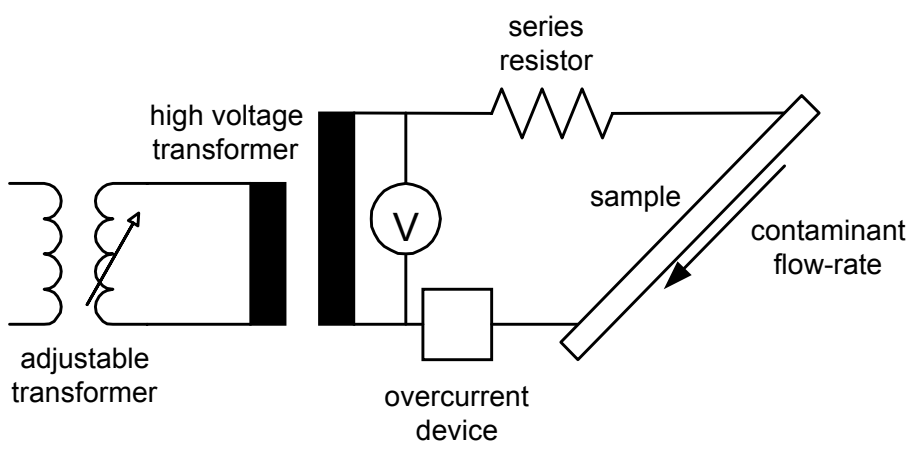

Figure 1 Schematic circuit diagram of Inclined-Plane Tracking 
LC flows along the sample surface. The level of LC significantly depends on the test parameters of IPT. These parameters value shall be strictly followed in accordance to the standard in order for the test to be successfully conducted. Improper values of IPT parameters used could greatly influence the grade of the IPT test [9].

\subsection{SYSTEM DEVELOPMENT}

The system for evaluating the surface tracking and erosion test along with the on-line LC monitoring system is shown in Figure 2. The whole system is placed inside a Faraday cage to avoid any outside noise or disturbance that could affect the test measurements. All the high voltage equi pments used and the Faraday cage are properly grounded for safety purpose.

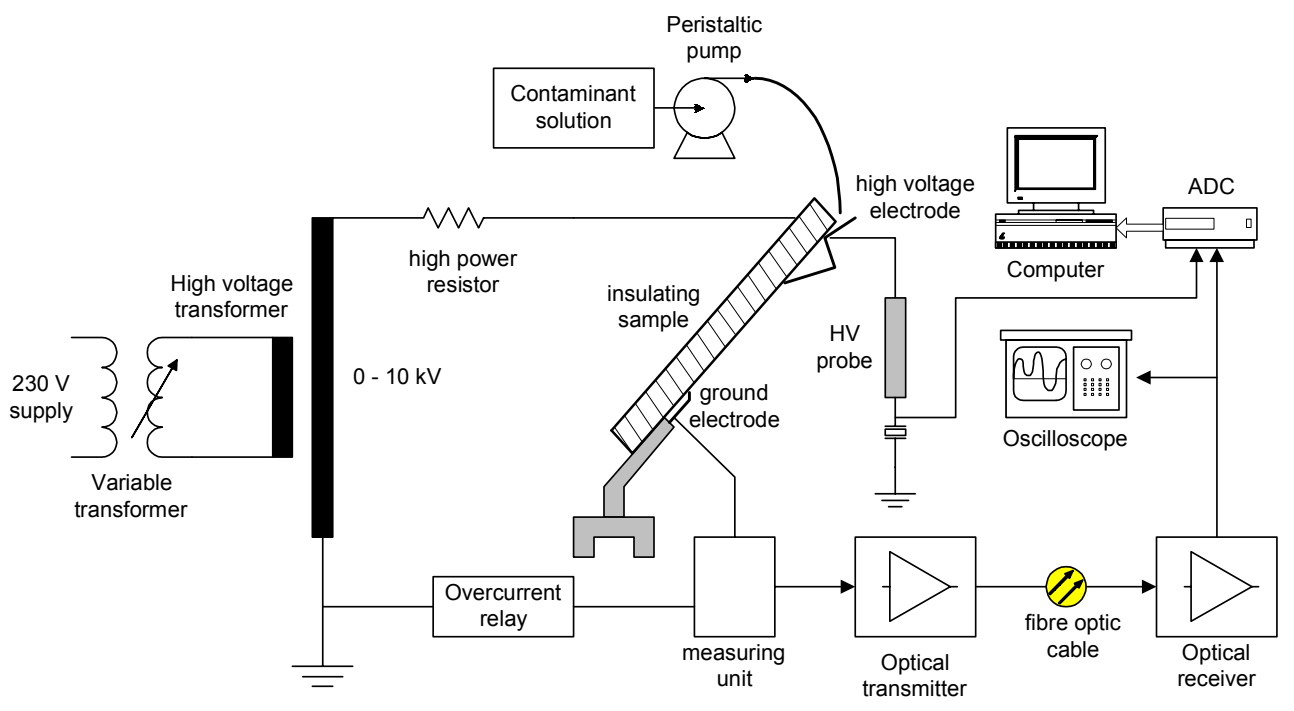

Figure 2 Surface Tracking and Erosion Test System with on-line LC monitoring system

\subsection{Hardware Set-up and Test Procedure}

A $1.0 \mathrm{kVA}, 0$ to $10 \mathrm{kV}$ high voltage transformer is used to supply a high voltage stress across a contaminated sample via a high power $200 \mathrm{~W}(1 \mathrm{k}-50 \mathrm{~kW})$ resistor. The sample is wet-contaminated by flowing down continuously the contaminant solution on top of the sample through a PVC tube. A peristaltic pump is used to flow the contaminant solution at very low flow-rate according to the standard procedure. The contaminant electrolyte contains $0.1 \%$ by mass of ammonium chloride solution with Triton X-100 non-ionic wetting agent. The resistivity of the contaminant solution is maintained between 370 and $400 \mathrm{~W}-\mathrm{cm}$. 
The slab-shaped sample with a dimension of $120 \times 50 \times 6 \mathrm{~mm}$ is mounted with the flat test surface on the underside, at an angle of $45^{\circ}$ from the horizontal with the stainless steel electrodes $50 \mathrm{~mm}$ apart. All electrodes, fixtures and assembly elements associated with the electrodes must be made of stainless steel. In order to get the proper flowing of contaminant solution, eight layers of filter paper are clamped between the top electrode and the sample. For safety purposes, over current devices such as a relay or a fuse is installed in series with the sample. This over current device will operate when $60 \mathrm{~mA}$ or more current has persisted in the high voltage circuit for 2 seconds.

The preferred test voltage depends on the contaminant flow-rate as well as the series resistor on the high voltage side. The IPT parameters values are suggested according to the following parameter relationship which is developed using dimensional analysis technique based on the magnitude of LC flows on the insulator surface [10].

$$
\frac{V a}{l}=\frac{\sqrt{R^{3} q p}}{\rho}+0.4
$$

where, $V a$ : applied voltage $(\mathrm{kV}), l$ : electrodes distance $(\mathrm{cm}), R$ : series resistor $(\mathrm{kW})$, $q$ : contaminant flow-rate (ml/min), $p$ : environmental pressure (bar), $r$ : contaminant solution resistivity $(\mathrm{W}-\mathrm{cm})$.

The test is successfully conducted when the effective scintillation is observed, which means the existence of small yellow to white arc just above the teeth of the lower electrode. This arc appears within a few minutes of applying the voltage.

\subsection{LC MONITORING SYSTEM}

An LC data acquisition system is developed to analyse the LC flowing on the sample surface under laboratory conditions. It consists of three parts;

(1) Measuring Unit

(2) Signal Conditioning

(3) Analog to Digital Converter (ADC) with personal computer

The measuring unit is a component that is used to capture the LC that flows along the sample surface. It is done by measuring the voltage drop across a resistor, which is connected in series with the ground electrode. The resistor value can be changed from $50 \mathrm{~W}$ to $1 \mathrm{~kW}$, depending on the LC level. This unit is also equipped with a protective circuit to protect the measurement system against occasional flashover during the test. The protective circuit consists of a $63 \mathrm{~mA}$ fuse, which is connected in series with ground electrode and parallel connection of a gas discharge tube (with a few nanoseconds operation) and a back-to-back $12 \mathrm{~V}$ Zenner diode. 
For a very small LC, the amplifier is developed to amplify the signal. The amplified signal is sent to a remote measuring system via a fibre optic cable. The use of an optical transceiver is to reduce the signal attenuation during transmission of the LC signal from the test system to the measurement system. A high power infrared photo emitter and a photo detector are used in the optical transceiver system. The highspeed photo emitter provides users with a universal LED emitter designed primarily for maximising the applications of polymer optical fibre. It also launches good levels of power into other multimode fibre types, and the fast response time makes it suitable for data rates up to $10 \mathrm{MB} / \mathrm{s}$.

The LC signal is analysed by a computer with the help of an external analog-todigital converter (ADC). A 12 bits, 2 channels $\mathrm{ADC}$ with $3 \mathrm{MS} / \mathrm{s}$ sampling rate manufactured by Pico Technology is used. This ADC has large $32 \mathrm{k}$ waveform buffers, so it is possible to capture complex signals and then expand areas of interest to show fine detail. The program based on the LabVIEW package, is written to communicate with the $\mathrm{ADC}$ to sort out the $\mathrm{LC}$ based on the magnitude and calculate the cumulative charge. In addition, the discrete Fourier Transform analysis of the LC waveform is performed on-line and its normalised harmonic components are sorted out.

For experimentation purposes, the LabVIEW program provides a variable sampling rate that can be chosen from. A faster sampling rate provides a more accurate reconstruction of the $\mathrm{LC}$ waveform for integration and lessens the degree of inaccuracy of the integration method. Besides the capability for on-line monitoring, a data storage feature is also added to the system to allow for future analysis.

\subsection{SOFTWARE DEVELOPMENT}

The graphical results of the LC signal are developed using LabVIEW software. LabVIEW is a program development environment, much like modern $\mathrm{C}$ or BASIC development environments, and uses a graphical programming language, $\mathrm{G}$, to create programs in block diagram forms. LabVIEW programs are called virtual instruments (VIs) because their appearance and operation can imitate actual instruments. A VI consists of an interactive user interface, a dataflow diagram that serves as the source code, and icon connections that allow the VI to be called from higher level VIs. More specifically, VIs are structured as follows [11];

- The interactive user interface of a VI is called the front panel. The data is entered and then the result is viewed on the computer screen in this front panel.

- The VI receives instructions from a block diagram. The block diagram is a pictorial solution to a programming problem, and it is also the source code for the VI.

- VIs are hierarchical and modular, and can use them as top-level programs, or as subprograms within other programs. 


\subsection{SIGNAL ANALYSIS}

The samples of a signal (discrete-time) obtained from the ADC constitute the time domain representation of the signal. This representation gives the amplitudes of the signal at the instants of time during which it had been sampled. The time domain representation (sample values) of a signal is converted into frequency domain representation by means of an algorithm known as discrete Fourier Transform (DFT), which is given by equation $[12,13]$;

$$
X(k)=\sum_{n=0}^{N-1} x(n) \exp \left(\frac{-j 2 \pi n k}{N}\right)
$$

for $(k=0,1,2, \ldots \ldots ., N-1)$, where $N$ is a number of samples and $x(n)$ is a sampled signal. The signal is sampled at a sampling rate of $f s \mathrm{~Hz}$. The time interval between the samples (ie. sampling interval) is $\mathrm{D} t$, where;

$$
\Delta t=\frac{1}{f_{s}}
$$

The frequency spacing between the components of $X$ in the frequency domain is given as follows;

$$
\Delta f=\frac{f_{s}}{N}=\frac{1}{N \Delta t}
$$

$\Delta f$ is also known as the frequency resolution. To increase frequency resolution (smaller $\Delta f$ ), the number of samples $N$ (with $f_{s}$ constant) is increased or decreased the sampling frequency $f_{s}$ (with $N$ constant). Both $N$ and $f_{s}$ can be varied from the front panel of the LabVIEW program.

In the discrete-time system, the finite sampling record results in a truncated waveform that has different spectral characteristics from the original continuous-time signal. These discontinuities produce leakage of spectral information, resulting in a discrete-time spectrum that is a smeared version of the original continuous-time spectrum. In order to improve the spectral characteristics of a sampled signal, the smoothing windows are applied to minimise the transition edges of the truncated waveforms to reduce the spectral leakage $[11,12,13]$. The smoothing windows act like predefined, narrowband lowpass filters. In this application, the input signal is windowed by passing it through one of the window VI component. The windowed signal is then passed to a DFTbased VI for frequency-domain display and analysis.

The front panel of the graphical user interface (GUI) of the developed program is shown in Figure 3. This illustration of the GUI collects the user input and displays the program output that the measurement system provides. The block diagram has a multi-frame sequence structure, which consists of more sub-diagrams, or frames, that 


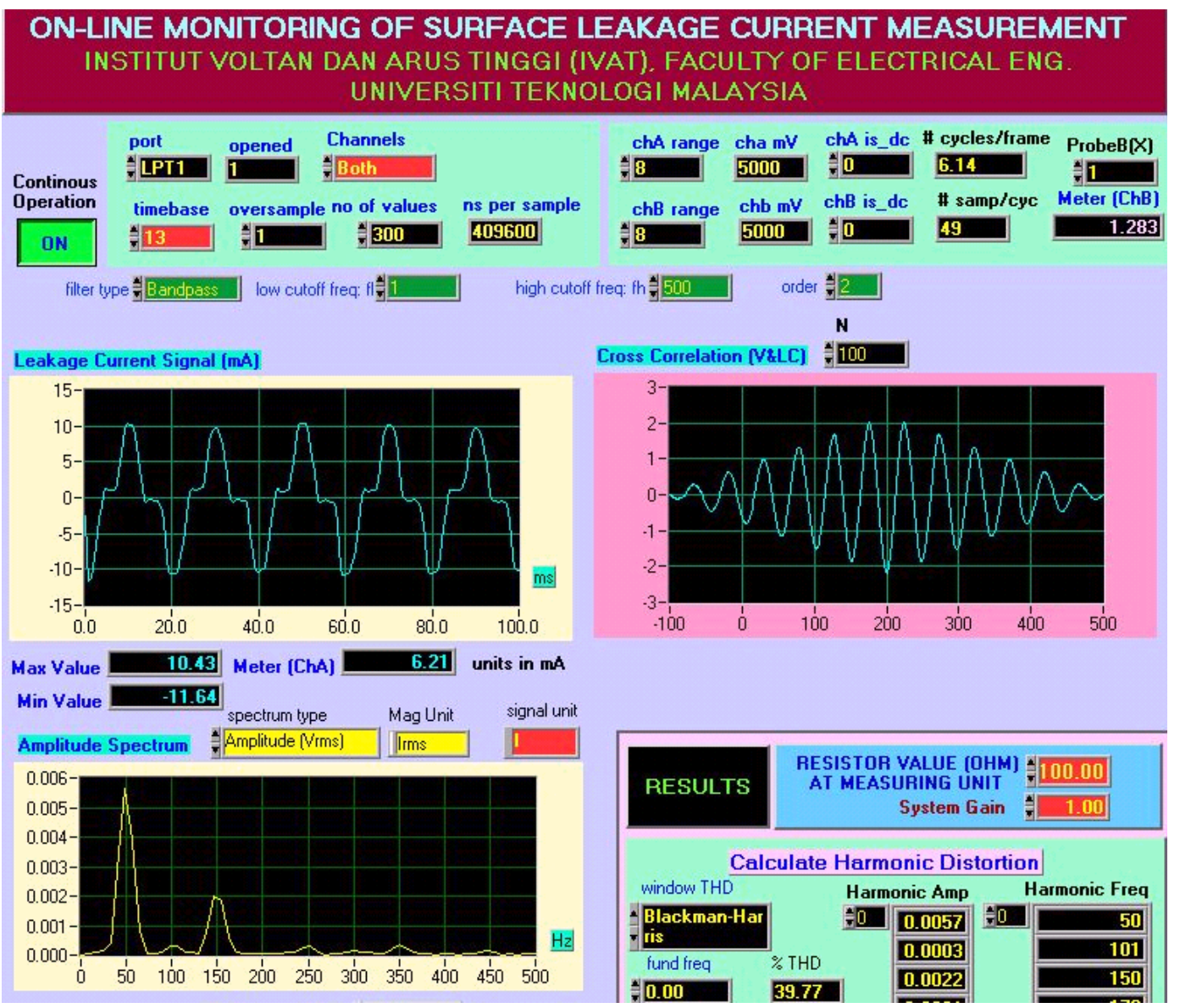

Figure 3 LabVIEW front panel

execute sequentially. Each frame indicates the process of data calculation for the required output. Figure 4 illustrates a part of the sub-diagrams in the block diagram for calculating the LC properties. The block diagram contains the graphical source code of the virtual instrument program that controls and performs functions on the inputs and outputs that has been created on the front panel.

\subsection{RESULTS AND DISCUSSIONS}

The effectiveness and reliability of the on-line monitoring system is tested by conducting an experiment according to the IPT method. A thermoplastic elastomer (consists of LLDPE and natural rubber) is used for testing the influence of hydrophobicity on the $\mathrm{LC}$ behavior. A $2.5 \mathrm{kV}$ voltage is applied across the sample, and a $0.1 \%$ ammonium chloride solution is used as a contamination agent. A $10 \mathrm{~kW}$ resistor of the IPT system is used in series connection for current limiting. The resistivity of the contaminant solution is about $385 \mathrm{~W}-\mathrm{cm}$ and flows at the rate of $0.15 \mathrm{ml} / \mathrm{min}$. 


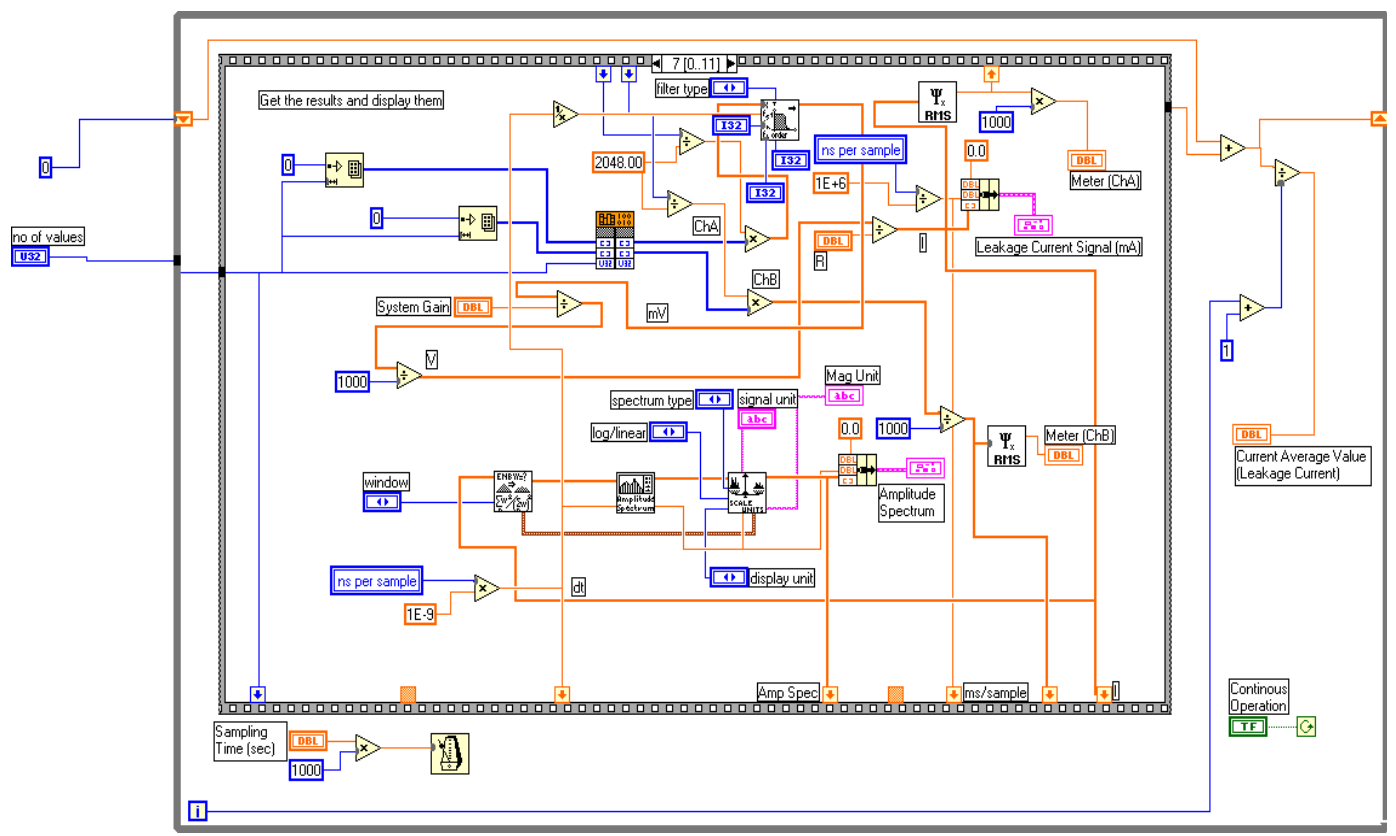

(a) Measurement of LC and displays waveform and frequency spectrum

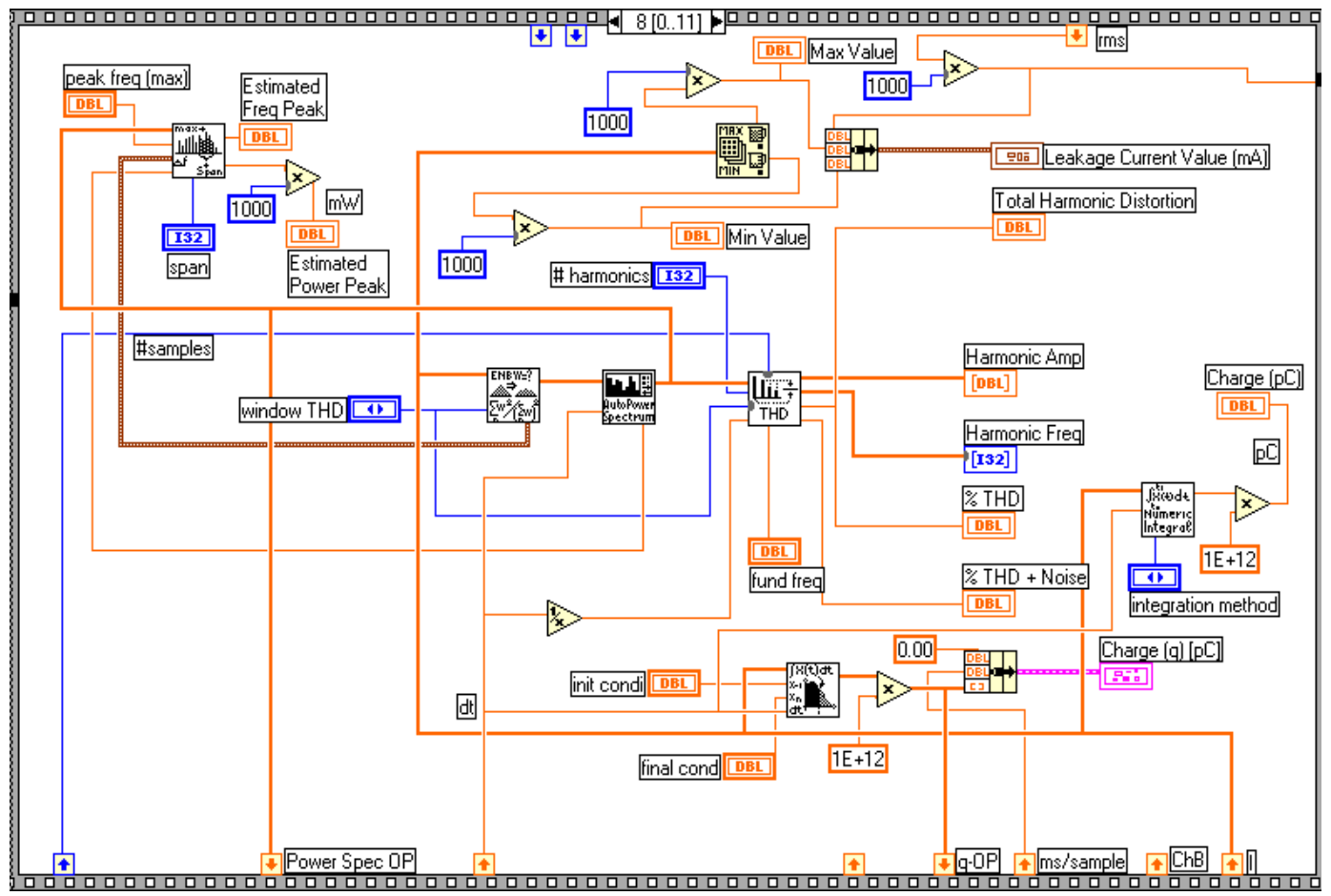

(b) Measurement of LC harmonic components, total charge and power

Figure 4 Sub-diagrams of VI block diagram for calculating LC properties 
Different stages of the LC behavior are recorded from the experiment, as illustrated in Figure 5. For the insulator that preserves the hydrophobic properties best, its LC waveform is very small and practically not detectable. But, when the insulator surface is completely wet, the $\mathrm{LC}$ appears to be sinusoidal and resistive with higher magnitude as shown in Figure 5.1(a). Almost no harmonic components are recorded as indicated in Figure 5.1(b). The total harmonic distortion (THD) of the waveform is about 1.67\% only. The value of the $T H D$ is calculated from the frequency spectrum using the following equation [14];

$$
\% T H D=\frac{100 \sqrt{A\left(I_{2}\right)^{2}+A\left(I_{3}\right)^{2}+\cdots+A\left(I_{N}\right)^{2}}}{A\left(I_{1}\right)}
$$

where, $A\left(I_{1}\right)$ : amplitude of the fundamental component, $A\left(I_{N}\right)$ : amplitude of the $N$ th harmonic and $N$ is the number of harmonics.

The cases in which the hydrophobic properties are either partially lost or weak dryband activities started, the LC patterns could be resistive and non-linear as depicted in Figure 5.2(a). The waveform becomes slightly triangular-shaped and has a little distortion at the crest of the waveform due to corona effect [15]. The frequency spectrum of the waveform in Figure 5.2(b) indicates slightly increase in the harmonic components with a $T H D$ of $8.04 \%$.

The waveform pattern in Figure 5.3(a) indicates the existence of several and random short discharges. These discharges seem to be "thinner" and much less energetic, and would only occur in the very early stages of wetting. The discharges move rapidly from one location to another location without causing any degradation. This condition makes the discharge current waveform appears as a series of pulses with nonsymmetrical pattern, and hence reflected by the high value of the THD, which is recorded as 109.48\%. The frequency spectrum in Figure 5.3(b) shows all the harmonic components due to the unsymmetrical waveform.

In the presence of intermittent, strong as well as continuous local arc, the recorded LC waveform is shown in Figure 5.4(a) with a THD of 57.5\%. The stable and continuous discharge results in the waveform pattern to be symmetry and contains odd harmonic components only as depicted in Figure 5.4(b). This LC is resistive in character and in phase with the applied voltage as shown in Figure 6 . The current can be detected only when the power frequency energizing voltage exceeds a certain positive or negative voltage threshold. At the instant where the LC is conducted, the voltage suddenly drops to about $400-500 \mathrm{~V}$. This voltage is the discharge voltage at the dry-band gap. During this phenomenon, it is observed that the electrical discharge stay rooted in a particular spot much longer and degradation of the insulating sample is initiated. It has been reported that discharge duration of more than 1 second can initiate the degradation of the sample [16]. This localized and persistent discharge activity can raise the temperature to cause degradation. 


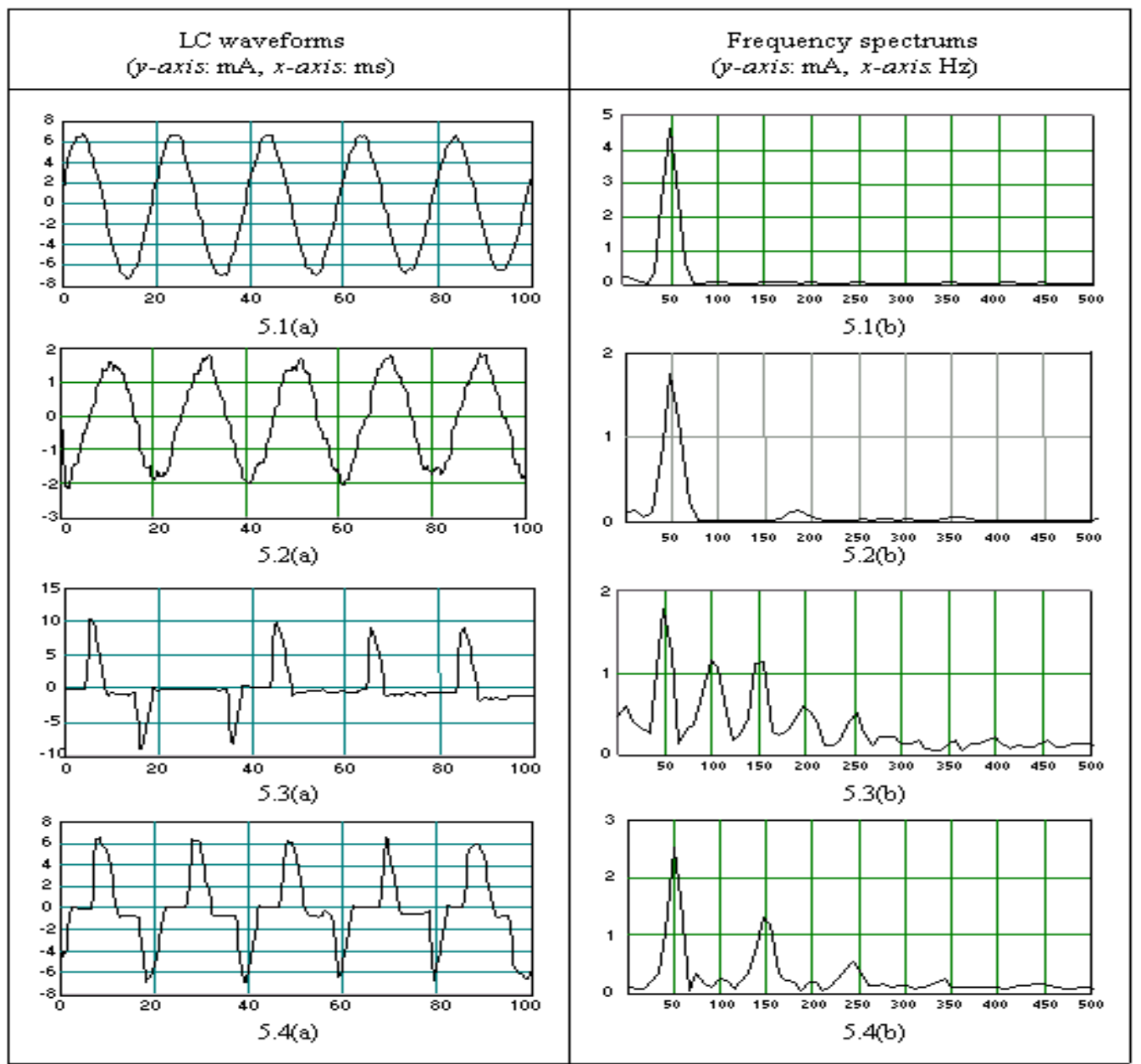

Figure 5 Typical LC waveforms and their spectral frequency

\subsection{CONCLUSION}

A practical on-line monitoring system at a reasonable cost has been designed and evaluated for surface leakage current under Inclined-Plane Test method. The use of such measuring technique permits a better understanding on the performance study of the insulator materials. The higher the total harmonic distortion of the leakage current waveform, the higher the probability of hydrophobic properties lost in the insulating materials. In addition, the characteristics of surface discharge have significantly influenced the on set of material degradation. The results suggest that the combination of leakage current magnitude and waveshapes analysis can provide a reliable indication on the status of insulating materials. The feature of data storage in the designed system could facilitate the researchers to extend the study for further analysis without conducting the test again. 


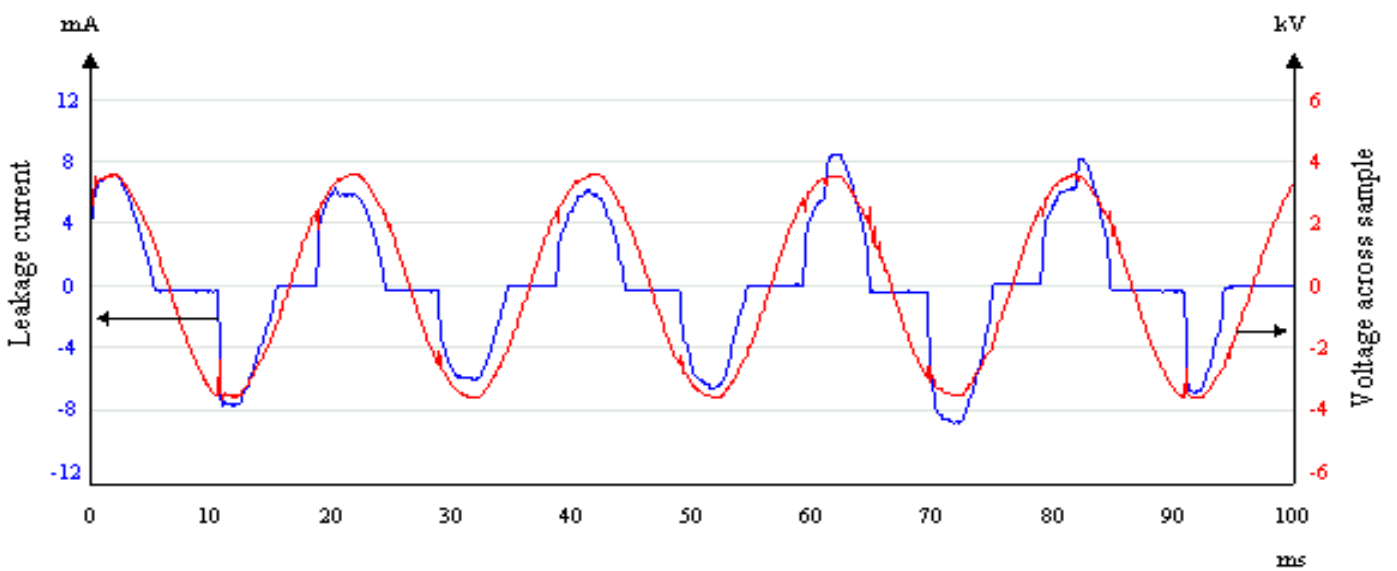

Figure 6 LC and applied voltage waveforms during continuous discharge

\section{REFERENCES}

[1] Hackam, R. 1999. Outdoor HV Composite Polymeric Insulators. IEEE Trans. On Dielectrics and Electrical Insulation. 6(5): 557-585.

[2] Barsch, R., H.J.Jahn, F. Lambrecht, F. Schmuck. 1999. Test Methods for Polymeric Insulating Materials for Outdoor HV Insulations. IEEE Trans. On Dielectric and Electrical Insulation. 6(5): 668-675.

[3] Fernando, M.A.R.M., S. M. Gubanski. 1999. Leakage Currents on Non-Ceramic Insulators and Materials. IEEE Trans. On Dielectrics and Electrical Insulation. 6(5): 660-667.

[4] Gorur, R. et.al. 1997. A Rapid Test Method for Evaluating the Tracking and Erosion Resistance of Polymeric Outdoor Insulating Materials. IEEE Conf. On Electrical Insulation and Dielectric Phenomena. Minneapolis, USA, pp. 402-405.

[5] Tjokrodi ponto, W. et al. 1997. Analysis of Visual Discharge Activities Along Polymer Insulators. Int. Conf. On Electrical Insulation and Dielectric Phenomena. Minneapolis, USA. 386-389.

[6] Varlow, B. R. 1996. Surface Insulation Testing Methods. The Institution of Electrical Engineers. London. 2(1-4).

[7] Suda, T. 2001. Frequency Characteristics of Leakage Current Waveforms of An Artificially Polluted Suspension Insulator. IEEE Trans. On Dielectric and Electrical Insulation. 8(4): 705-709.

[8] IEC 587. 1984. Standard Methods for Evaluating Resistance to Tracking and Erosion of Electrical Insulating Materials Used Under Severe Ambient Conditions. British Standard (BS).

[9] Xiaoqing, G. 1988. Factors which may affect the inclined plane classification of materials. Int. Conf. On Properties and Appplications of Dielectric Materials. 1: 233-236.

[10] Piah, M.A.M., A. Darus. 2002. Mathematical Analysis for Parameters Variation in Inclined-Plane Tracking Test Method. IEEE Region 10 Technical Conference. Beijing, China. 3: 1877-1880.

[11] LabVIEW User Manual, 1998. National Instruments.

[12] Soliman, S.S., M.D. Srinath. 1998. Continuous and Discrete Signals and System. New Jersey: Prentice Hall.

[13] Hayes, M.H. 1999. Digital Signal Processing. USA: McGraw Hill.

[14] LabVIEW User Manual. 1998. Spectrum Analysis and Measurement. Chp. 15, National Instruments.

[15] Fernando, M.A.R.M., S. M. Gubanski. 1999. Leakage Current Patterns on Contaminated Polymeric Surfaces. IEEE Trans. On Dielectrics and Electrical Insulation. 6(5): 688-694.

[16] Gorur, R. S. et.al. 1997. A Laboratory Test for Tracking and Erosion Resistance of HV Outdoor Insulation. IEEE Trans. On Dielectrics and Electrical Insulation. 4(6): 767-774. 